\title{
Performance Attribution for Equity Portfolios
}

by Yang Lu and David Kane

\begin{abstract}
The pa package provides tools for conducting performance attribution for long-only, single currency equity portfolios. The package uses two methods: the Brinson-Hood-Beebower model (hereafter referred to as the Brinson model) and a regression-based analysis. The Brinson model takes an ANOVA-type approach and decomposes the active return of any portfolio into asset allocation, stock selection, and interaction effect. The regression-based analysis utilizes estimated coefficients, based on a regression model, to attribute active return to different factors.
\end{abstract}

\section{Introduction}

Almost all portfolio managers measure performance with reference to a benchmark. The difference in return between a portfolio and the benchmark is its active return. Performance attribution decomposes the active return. The two most common approaches are the Brinson model (Brinson et al., 1986) and a regression-based analysis (Grinold, 2006).

Portfolio managers use different variations of the two models to assess the performance of their portfolios. Managers of fixed income portfolios include yield-curve movements in the model (Lord, 1997) while equity managers who focus on the effect of currency movements use variations of the Brinson model to incorporate "local risk premium" (Singer and Karnosky, 1995). In contrast, in this paper we focus on attribution models for long-only equity portfolios without considering any currency effect. $^{1}$

The pa package provides tools for conducting both methods for long-only, single currency equity portfolios. ${ }^{2}$ The Brinson model takes an ANOVA-type approach and decomposes the active return of any portfolio into asset allocation, stock selection, and interaction effects. The regression-based analysis utilizes estimated coefficients from a linear model to estimate the contributions from different factors.

\section{Data}

We demonstrate the use of the pa package with a series of examples based on data from MSCI Barra's Global Equity Model II (GEM2). ${ }^{3}$ The original data set contains selected attributes such as industry, size, country, and various style factors for a universe of approximately 48,000 securities on a monthly basis. For illustrative purposes, this article uses three modified versions of the original data set (year, quarter, and jan), each containing 3000 securities. The data frame, quarter, is a subset of year, containing the data of the first quarter. The data frame, jan, is a subset of quarter with the data from January, 2010.

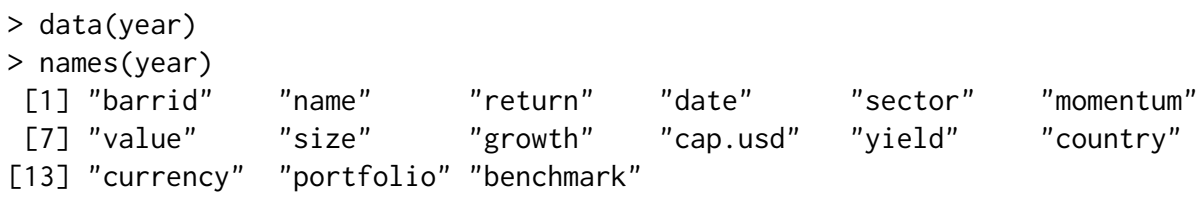

See ?year for information on the different variables. The top 200 securities, based on value scores, in January are selected as portfolio holdings and are held through December 2010 with monthly rebalances to maintain equal-weighting. The benchmark for this portfolio is defined as the largest 1000 securities based on size each month. The benchmark is cap-weighted.

Here is a sample of rows and columns from the data frame year:

\footnotetext{
${ }^{1}$ See Morningstar Inc (2009) for a comprehensive discussion of conventional attribution methods.

${ }^{2}$ There are several $R$ packages which provide related functionality: portfolio (Enos and Kane, 2006) enables users to analyze and implement equity portfolios; PerformanceAnalytics (Carl and Peterson, 2013) provides a collection of econometric functions for performance and risk analysis; the Rmetrics suite contains a collection of functions for computational finance (Rmetrics Association, 2013). Although PerformanceAnalytics and the Rmetrics suite provide a variety of tools, they do not provide for the attribution of returns using the Brinson Model.

${ }^{3}$ See www . msci . com and Menchero et al. (2008) for more information.
} 


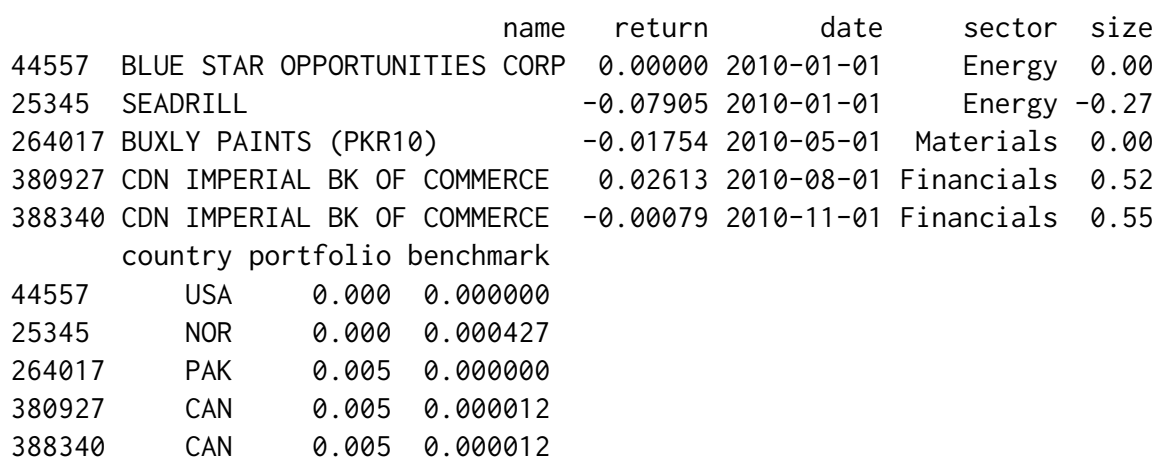

The portfolio has 200 equal-weighted holdings each month. The row for Canadian Imperial Bank of Commerce indicates that it is one of the 200 portfolio holdings with a weight of $0.5 \%$ in 2010 . Its return was $2.61 \%$ in August, and close to flat in November.

\section{Brinson model}

Consider an equity portfolio manager who uses the S\&P 500 as the benchmark. In a given month, she outperformed the S\&P by 3\%. Part of that performance was due to the fact that she allocated more weight of the portfolio to certain sectors that performed well. Call this the allocation effect. Part of her outperformance was due to the fact that some of the stocks she selected did better than their sector as a whole. Call this the selection effect. The residual can then be attributed to an interaction between allocation and selection - the interaction effect. The Brinson model provides mathematical definitions for these terms and methods for calculating them.

The example above uses sector as the classification scheme when calculating the allocation effect. But the same approach can work with any other variable which places each security into one, and only one, discrete category: country, industry, and so on. In fact, a similar approach can work with continuous variables that are split into discrete ranges: the highest quintile of market cap, the second highest quintile and so forth. For generality, we will use the term "category" to describe any classification scheme which places each security in one, and only one, category.

Notations:

- $w_{i}^{B}$ is the weight of security $i$ in the benchmark.

- $w_{i}^{P}$ is the weight of security $i$ in the portfolio.

- $W_{j}^{B}$ is the weight of category $j$ in the benchmark. $W_{j}^{B}=\sum w_{i}^{B}, i \in j$.

- $W_{j}^{P}$ is the weight of a category $j$ in the portfolio. $W_{j}^{P}=\sum w_{i}^{P}, i \in j$.

- The sum of the weight $w_{i}^{B}, w_{i}^{P}, W_{j}^{B}$, and $W_{j}^{P}$ is 1 , respectively.

- $r_{i}$ is the return of security $i$.

- $R_{j}^{B}$ is the return of a category $j$ in the benchmark. $R_{j}^{B}=\sum w_{i}^{B} r_{i}, i \in j$.

- $R_{j}^{P}$ is the return of a category $j$ in the portfolio. $R_{j}^{P}=\sum w_{i}^{P} r_{i}, i \in j$.

The return of a portfolio, $R_{P}$, can be calculated in two ways:

- On an individual security level by summing over $n$ stocks: $R_{P}=\sum_{i=1}^{n} w_{i}^{P} r_{i}$.

- On a category level by summing over $N$ categories: $R_{P}=\sum_{j=1}^{N} W_{j}^{P} R_{j}^{P}$.

Similar definitions apply to the return of the benchmark, $R_{B}$,

$$
R_{B}=\sum_{i=1}^{n} w_{i}^{B} r_{i}=\sum_{j=1}^{N} W_{j}^{B} R_{j}^{B}
$$

Active return of a portfolio, $R_{\text {active, }}$ is a performance measure of a portfolio relative to its benchmark. The two conventional measures of active return are arithmetic and geometric. The pa package implements the arithmetic measure of the active return for a single-period Brinson model because an arithmetic difference is more intuitive than a ratio over a single period.

The arithmetic active return of a portfolio, $R_{\text {active, }}$ is the portfolio return $R_{P}$ less the benchmark return $R_{B}$ : 


$$
R_{\text {active }}=R_{P}-R_{B} \text {. }
$$

Since the category weights of the portfolio are generally different from those of the benchmark, allocation plays a role in the active return, $R_{\text {active }}$. The same applies to stock selection effects. Within a given category, the portfolio and the benchmark will rarely have exactly the same holdings. Allocation effect $R_{\text {allocation }}$ and selection effect $R_{\text {selection }}$ over $N$ categories are defined as:

$$
R_{\text {allocation }}=\sum_{j=1}^{N}\left(W_{j}^{P}-W_{j}^{B}\right) R_{j}^{B}
$$

and

$$
R_{\text {selection }}=\sum_{j=1}^{N} W_{j}^{B}\left(R_{j}^{P}-R_{j}^{B}\right) .
$$

The intuition behind the allocation effect is that a portfolio would produce different returns with different allocation schemes $\left(W_{j}^{P}\right.$ vs. $\left.W_{j}^{B}\right)$ while having the same stock selection and thus the same return $\left(R_{j}^{B}\right)$ for each category. The difference between the two returns, caused by the allocation scheme, is called the allocation effect $\left(R_{\text {allocation }}\right)$. Similarly, two different returns can be produced when two portfolios have the same allocation $\left(W_{j}^{B}\right)$ yet dissimilar returns due to differences in stock selection within each category $\left(R_{j}^{p}\right.$ vs. $\left.R_{j}^{B}\right)$. This difference is the selection effect $\left(R_{\text {selection }}\right)$.

Interaction effect $\left(R_{\text {interaction }}\right)$ is the result of subtracting return due to allocation $R_{\text {allocation }}$ and return due to selection $R_{\text {selection }}$ from the active return $R_{\text {active }}$ :

$$
R_{\text {interaction }}=R_{\text {active }}-R_{\text {allocation }}-R_{\text {selection }} .
$$

The Brinson model allows portfolio managers to analyze the active return of a portfolio using any attribute of a security, such as country or sector. Unfortunately, it is very hard to expand the analysis beyond two categories. As the number of categories increases, the number of terms to be included in the Brinson model grows exponentially; this procedure is thus subject to the curse of dimensionality. To some extent, the regression-based model detailed later ameliorates this problem.

\section{Brinson tools}

Brinson analysis is run by calling the function brinson to produce an object of class "brinson".

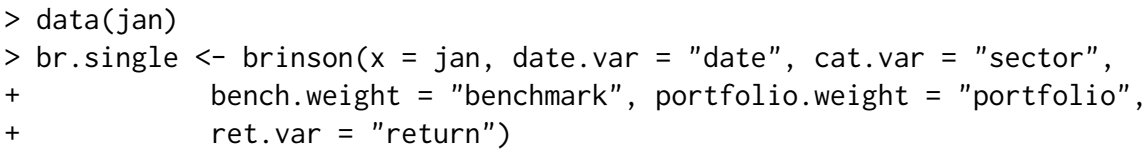

The data frame, jan, contains all the information necessary to conduct a single-period Brinson analysis. date.var, cat.var, and return identify the columns containing the date, the factor to be analyzed, and the return variable, respectively. bench. weight and portfolio. weight specify the name of the benchmark weight column and that of the portfolio weight column in the data frame.

Calling summary on the resulting object br. single of class "brinson" reports essential information about the input portfolio (including the number of securities in the portfolio and the benchmark as well as sector exposures) and the results of the Brinson analysis (both by sector and aggregate).

$\begin{array}{lrrr}\text { > summary(br.single) } & \\ \text { Period: } & & \\ \text { Methodology: } & & \\ \text { Brin } & & \\ \text { Securities in the portfolio: } & 200 \\ \text { Securities in the benchmark: } & 1000 \\ & & & \\ \text { Exposures } & & & \\ & & \\ \text { Energy } & 0.085 & 0.2782 & -0.19319 \\ \text { Materials } & 0.070 & 0.0277 & 0.04230 \\ \text { Industrials } & 0.045 & 0.0330 & 0.01201 \\ \text { ConDiscre } & 0.050 & 0.0188 & 0.03124 \\ \text { ConStaples } & 0.030 & 0.0148 & 0.01518 \\ \text { HealthCare } & 0.015 & 0.0608 & -0.04576\end{array}$




$\begin{array}{lrrr}\text { Financials } & 0.370 & 0.2979 & 0.07215 \\ \text { InfoTech } & 0.005 & 0.0129 & -0.00787 \\ \text { TeleSvcs } & 0.300 & 0.1921 & 0.10792 \\ \text { Utilities } & 0.030 & 0.0640 & -0.03399\end{array}$

Returns

\$'Attribution by category in bps'

Allocation Selection Interaction

$\begin{array}{lrrr}\text { Energy } & 110.934 & -37.52 & 26.059 \\ \text { Materials } & -41.534 & 0.48 & 0.734 \\ \text { Industrials } & 0.361 & 1.30 & 0.473 \\ \text { ConDiscre } & -28.688 & -4.23 & -7.044 \\ \text { ConStaples } & 5.467 & -3.59 & -3.673 \\ \text { HealthCare } & -6.692 & -4.07 & 3.063 \\ \text { Financials } & -43.998 & 70.13 & 16.988 \\ \text { InfoTech } & -3.255 & -5.32 & 3.255 \\ \text { TeleSvcs } & -23.106 & 41.55 & 23.348 \\ \text { Utilities } & 16.544 & 83.03 & -44.108 \\ \text { Total } & -13.966 & 141.77 & 19.095\end{array}$

\$Aggregate

$\begin{array}{lr} & 2010-01-01 \\ \text { Allocation Effect } & -0.00140 \\ \text { Selection Effect } & 0.01418 \\ \text { Interaction Effect } & 0.00191 \\ \text { Active Return } & 0.01469\end{array}$

The br. single summary shows that the active return of the portfolio, in January, 2010 was 1.47\%. This return can be decomposed into allocation effect $(-0.14 \%)$, selection effect $(1.42 \%)$, and interaction effect $(0.19 \%)$.

$>\operatorname{plot}(\mathrm{br}$. single, $\operatorname{var}=$ "sector", type $=$ "return")

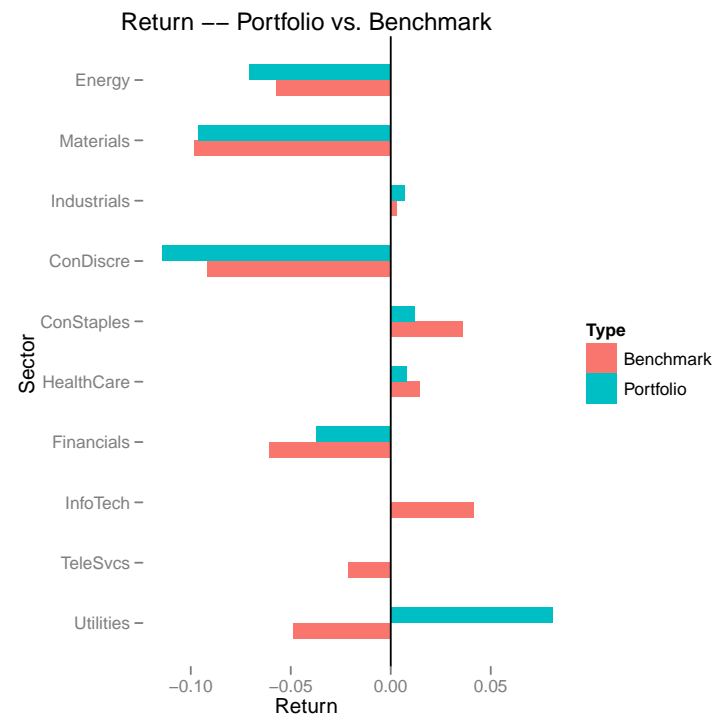

Figure 1: Sector Return.

Figure 1 is a visual representation of the return of both the portfolio and the benchmark sector by sector in January, 2010. Utilities was the sector with the highest active return in the portfolio.

To obtain Brinson attribution on a multi-period data set, one calculates allocation, selection and interaction within each period and aggregates them across time. There are three methods for this - arithmetic, geometric, and optimized linking (Menchero, 2000). The arithmetic attribution model calculates active return and contributions due to allocation, selection, and interaction in each period and sums them over multiple periods.

In practice, analyzing a single-period portfolio is meaningless as portfolio managers and their clients are more interested in the performance of a portfolio over multiple periods. To apply the 
Brinson model over time, we can use the function brinson and input a multi-period data set (for instance, quarter) as shown below.

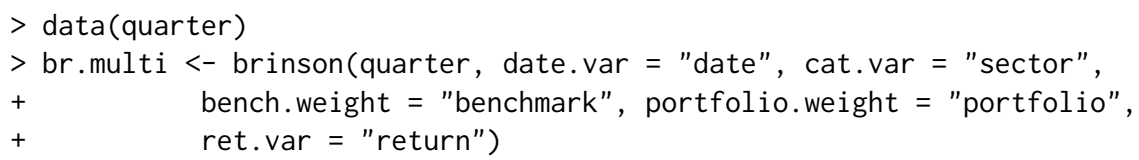

The object br.multi of class "brinsonMulti" is an example of a multi-period Brinson analysis.

> exposure(br.multi, var = "size")
\$Portfolio
$\begin{array}{lrrr}\text { 2010-01-01 } & 2010-02-01 & 2010-03-01 \\ \text { Low } & 0.140 & 0.140 & 0.155 \\ 2 & 0.050 & 0.070 & 0.045 \\ 3 & 0.175 & 0.145 & 0.155 \\ 4 & 0.235 & 0.245 & 0.240 \\ \text { High } & 0.400 & 0.400 & 0.405\end{array}$

\$Benchmark

$\begin{array}{lrrr} & 2010-01-01 & 2010-02-01 & 2010-03-01 \\ \text { Low } & 0.0681 & 0.0568 & 0.0628 \\ 2 & 0.0122 & 0.0225 & 0.0170 \\ 3 & 0.1260 & 0.1375 & 0.1140 \\ 4 & 0.2520 & 0.2457 & 0.2506 \\ \text { High } & 0.5417 & 0.5374 & 0.5557\end{array}$

\$Diff

$\begin{array}{lrrr} & 2010-01-01 & 2010-02-01 & 2010-03-01 \\ \text { Low } & 0.0719 & 0.083157 & 0.0922 \\ 2 & 0.0378 & 0.047456 & 0.0280 \\ 3 & 0.0490 & 0.007490 & 0.0410 \\ 4 & -0.0170 & -0.000719 & -0.0106 \\ \text { High } & -0.1417 & -0.137385 & -0.1507\end{array}$

The exposure method on the br.multi object shows the exposure of the portfolio and the benchmark, and their difference based on a user-specified variable. Here, it shows the exposure on size. We can see that the portfolio overweights the benchmark in the lowest quintile in size and underweights in the highest quintile.

$\begin{array}{lrrr}\begin{array}{l}\text { returns(br.multi, type }=\text { "arithmetic") } \\ \text { \$Raw }\end{array} & & & \\ & 2010-01-01 & 2010-02-01 & 2010-03-01 \\ \text { Allocation } & -0.0014 & 0.0062 & 0.0047 \\ \text { Selection } & 0.0142 & 0.0173 & -0.0154 \\ \text { Interaction } & 0.0019 & -0.0072 & -0.0089 \\ \text { Active Return } & 0.0147 & 0.0163 & -0.0196\end{array}$

$\begin{array}{lr}\text { \$Aggregate } & \\ & 2010-01-01, \\ \text { Allocation } & 0.00905 \\ \text { Selection } & 0.0160 \\ \text { Interaction } & -0.0142 \\ \text { Active Return } & 0.0114\end{array}$

The returns method shows the results of the Brinson analysis applied to the data from January, 2010 through March, 2010. The first portion of the returns output shows the Brinson attribution in individual periods. The second portion shows the aggregate attribution results. The portfolio formed by top 200 value securities in January had an active return of $1.14 \%$ over the first quarter of 2010 . The allocation and the selection effects contributed $0.95 \%$ and $1.6 \%$ respectively; the interaction effect decreased returns by $1.42 \%$.

\section{Regression}

One advantage of a regression-based approach is that such analysis allows one to define their own attribution model by easily incorporating multiple variables in the regression formula. These variables 
can be either discrete or continuous.

Suppose a portfolio manager wants to find out how much each of the value, growth, and momentum scores of her holdings contributes to the overall performance of the portfolio. Consider the following linear regression without the intercept term based on a single-period portfolio of $n$ securities with $k$ different variables:

$$
\mathbf{r}_{n}=\mathbf{X}_{n, k} \mathbf{f}_{k}+\mathbf{u}_{n}
$$

where

- $\mathbf{r}_{n}$ is a column vector of length $n$. Each element in $\mathbf{r}_{n}$ represents the return of a security in the portfolio.

- $\mathbf{X}_{n, k}$ is an $n$ by $k$ matrix. Each row represents $k$ attributes of a security. There are $n$ securities in the portfolio.

- $\mathbf{f}_{k}$ is a column vector of length $k$. The elements are the estimated coefficients from the regression. Each element represents the factor return of an attribute.

- $\mathbf{u}_{n}$ is a column vector of length $n$ with residuals from the regression.

In the case of this portfolio manager, suppose that she only has three holdings in her portfolio. $r_{3}$ is thus a 3 by 1 matrix with returns of all her three holdings. The matrix $\mathbf{X}_{3,3}$ records the score for each of the three factors (value, growth, and momentum) in each row. $\mathbf{f}_{3}$ contains the estimated coefficients of a regression $\mathbf{r}_{3}$ on $\mathrm{X}_{3,3}$.

The active exposure of each of the $k$ variables, $X_{i}, i \in k$, is expressed as

$$
X_{i}=\mathbf{w}_{\text {active }} / \mathbf{x}_{n, i},
$$

where $X_{i}$ is the value representing the active exposure of the attribute $i$ in the portfolio, $\mathbf{w}_{\text {active }}$ is a column vector of length $n$ containing the active weight of every security in the portfolio, and $\mathbf{x}_{n, i}$ is a column vector of length $n$ with attribute $i$ for all securities in the portfolio. Active weight of a security is defined as the difference between the portfolio weight of the security and its benchmark weight.

Using the example mentioned above, the active exposure of the attribute value, $X_{\text {value }}$ is the product of $\mathbf{w}_{\text {active }}$ (containing active weight of each of the three holdings) and $\mathbf{x}_{3}$ (containing value scores of the three holdings).

The contribution of a variable $i, R_{i}$, is thus the product of the factor returns for the variable $i, f_{i}$ and the active exposure of the variable $i, X_{i}$. That is,

$$
R_{i}=f_{i} X_{i}
$$

Continuing the example, the contribution of value is the product of $f_{\text {value }}$ (the estimated coefficient for value from the linear regression) and $X_{\text {value }}$ (the active exposure of value as shown above).

Therefore, the active return of the portfolio $R_{\text {active }}$ is the sum of contributions of all $k$ variables and the residual $u$ (a.k.a. the interaction effect),

$$
R_{\text {active }}=\sum_{i=1}^{k} R_{i}+u
$$

\begin{tabular}{|c|c|c|c|c|}
\hline Return & Name & Size & Value & Active_Weight \\
\hline 0.3 & A & 1.2 & 3.0 & 0.5 \\
\hline 0.4 & B & 2.0 & 2.0 & 0. \\
\hline 0.5 & C & 0.8 & 1.5 & -0.6 \\
\hline
\end{tabular}

For instance, a hypothetical portfolio has three holdings (A, B, and C), each of which has two attributes - size and value.

Following the procedure as mentioned, the factor returns for size and value are -0.0313 and -0.1250 . The active exposure of size is 0.32 and that of value is 0.80 . The active return of the portfolio is $-11 \%$ which can be decomposed into the contribution of size and that of value based on the regression model. Size contributes $1 \%$ of the negative active return of the portfolio and value causes the portfolio to lose the other $10.0 \%$.

\section{Regression tools}

The pa package provides tools to analyze both single-period and multi-period data frames. 


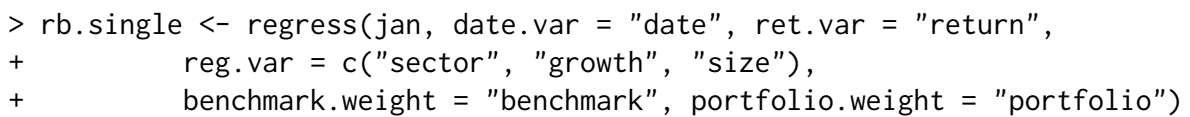

reg. var specifies the columns containing variables whose contributions are to be analyzed. Each of the reg. var input variables corresponds to a particular column in $\mathbf{X}_{n, k}$ from the aforementioned regression model. ret.var specifies the column in the data frame jan based on which $\mathbf{r}_{n}$ in the regression model is formed.

$\begin{array}{lrrr}> & \text { exposure(rb.single, var }=\text { "growth") } \\ & \text { Portfolio } & \text { Benchmark } & \text { Diff } \\ \text { Low } & 0.305 & 0.2032 & 0.1018 \\ 2 & 0.395 & 0.4225 & -0.0275 \\ 3 & 0.095 & 0.1297 & -0.0347 \\ 4 & 0.075 & 0.1664 & -0.0914 \\ \text { High } & 0.130 & 0.0783 & 0.0517\end{array}$

Calling exposure with a specified var yields information on the exposure of both the portfolio and the benchmark by that variable. If var is a continuous variable, for instance, growth, the exposure will be shown in 5 quantiles. Majority of the high value securities in the portfolio in January have relatively low growth scores.

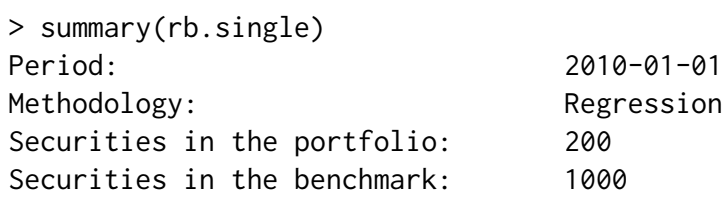

$\begin{array}{lr}\text { Returns } & \\ & 2010-01-01 \\ \text { sector } & 0.003189 \\ \text { growth } & 0.000504 \\ \text { size } & 0.002905 \\ \text { Residual } & 0.008092 \\ \text { Portfolio Return } & -0.029064 \\ \text { Benchmark Return } & -0.043753 \\ \text { Active Return } & 0.014689\end{array}$

The summary method shows the number of securities in the portfolio and the benchmark, and the contribution of each input variable according to the regression-based analysis. In this case, the portfolio made a loss of $2.91 \%$ and the benchmark lost $4.38 \%$. Therefore, the portfolio outperformed the benchmark by $1.47 \%$. sector, growth, and size contributed $0.32 \%, 0.05 \%$, and $0.29 \%$, respectively.

Regression-based analysis can be applied to a multi-period data frame by calling the same method regress. By typing the name of the object rb.multi directly, a short summary of the analysis is provided, showing the starting and ending period of the analysis, the methodology, and the average number of securities in both the portfolio and the benchmark.

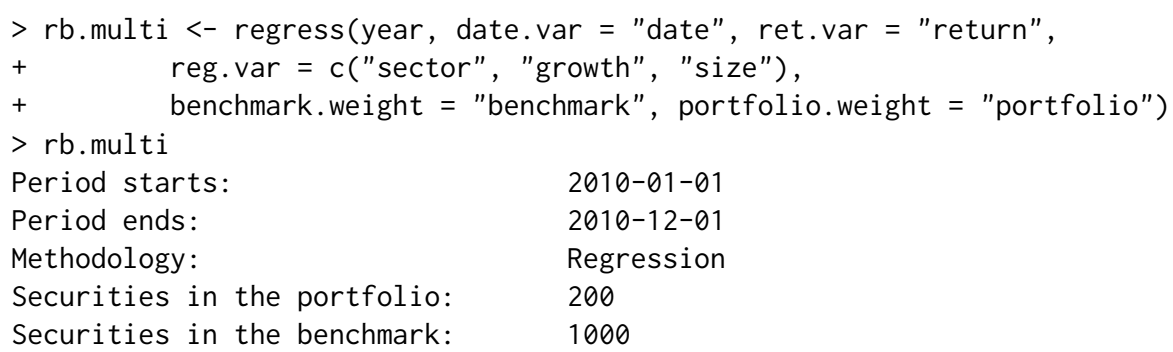

The regression-based summary shows that the contribution of each input variable in addition to the basic information on the portfolio. The summary suggests that the active return of the portfolio in year 2010 is $10.1 \%$. The Residual number indicates the contribution of the interaction among various variables including sector, growth, and size. Based on the regression model, size contributed to the lion's share of the active return.

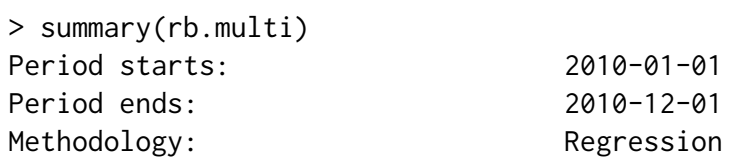


Avg securities in the portfolio: 200

Avg securities in the benchmark: 1000

Returns

\$Raw

$\begin{array}{lrrr} & 2010-01-01 & 2010-02-01 & 2010-03-01 \\ \text { sector } & 0.0032 & 0.0031 & 0.0002 \\ \text { growth } & 0.0005 & 0.0009 & -0.0001 \\ \text { size } & 0.0029 & 0.0295 & 0.0105 \\ \text { Residual } & 0.0081 & -0.0172 & -0.0302 \\ \text { Portfolio Return } & -0.0291 & 0.0192 & 0.0298 \\ \text { Benchmark Return } & -0.0438 & 0.0029 & 0.0494 \\ \text { Active Return } & 0.0147 & 0.0163 & -0.0196 \\ & 2010-04-01 & 2010-05-01 & 2010-06-01 \\ \text { sector } & 0.0016 & 0.0039 & 0.0070 \\ \text { growth } & 0.0001 & 0.0002 & 0.0004 \\ \text { size } & 0.0135 & 0.0037 & 0.0018 \\ \text { Residual } & -0.0040 & 0.0310 & 0.0183 \\ \text { Portfolio Return } & -0.0080 & -0.0381 & 0.0010 \\ \text { Benchmark Return } & -0.0192 & -0.0769 & -0.0266 \\ \text { Active Return } & 0.0113 & 0.0388 & 0.0276\end{array}$

$\begin{array}{llll}\text { Active Return } & 0.0113 & 0.0388 & 0.0276\end{array}$

$2010-07-01 \quad 2010-08-01 \quad 2010-09-01$

$\begin{array}{lrrr}\text { sector } & 0.0016 & 0.0047 & -0.0022 \\ \text { growth } & -0.0005 & 0.0005 & -0.0006 \\ \text { size } & 0.0064 & 0.0000 & 0.0096 \\ \text { Residual } & -0.0324 & 0.0173 & -0.0220 \\ \text { Portfolio Return } & 0.0515 & -0.0119 & 0.0393 \\ \text { Benchmark Return } & 0.0764 & -0.0344 & 0.0545 \\ \text { Active Return } & -0.0249 & 0.0225 & -0.0152 \\ & 2010-10-01 & 2010-11-01 & 2010-12-01 \\ \text { sector } & 0.0015 & -0.0044 & -0.0082 \\ \text { growth } & -0.0010 & -0.0004 & 0.0010 \\ \text { size } & 0.0022 & 0.0130 & 0.0056 \\ \text { Residual } & 0.0137 & 0.0175 & -0.0247 \\ \text { Portfolio Return } & 0.0414 & -0.0036 & 0.0260 \\ \text { Benchmark Return } & 0.0249 & -0.0293 & 0.0523 \\ \text { Active Return } & 0.0165 & 0.0257 & -0.0263\end{array}$

\$Aggregate

$2010-01-01,2010-12-01$

sector

0.0120

growth

0.0011

size

0.1030

Residual

$-0.0269$

Portfolio Return $\quad 0.1191$

Benchmark Return $\quad 0.0176$

Active Return $\quad 0.1015$

Figure 2 displays both the cumulative portfolio and benchmark returns from January, 2010 through December, 2010. It suggests that the portfolio, consisted of high value securities in January, consistently outperformed the benchmark in 2010. Outperformance in May and June helped the overall positive active return in 2010 to a large extent.

$>\operatorname{plot}($ rb.multi, var $=$ "sector", type $=$ "return")

\section{Conclusion}

In this paper, we describe two widely-used methods for performance attribution - the Brinson model and the regression-based approach, and provide a simple collection of tools to implement these two methods in $\mathrm{R}$ with the pa package. A comprehensive package, portfolio (Enos and Kane, 2006), provides facilities to calculate exposures and returns for equity portfolios. It is possible to use the pa package based on the output from the portfolio package. Further, the flexibility of R itself allows users to extend and modify these packages to suit their own needs and/or execute their preferred attribution 


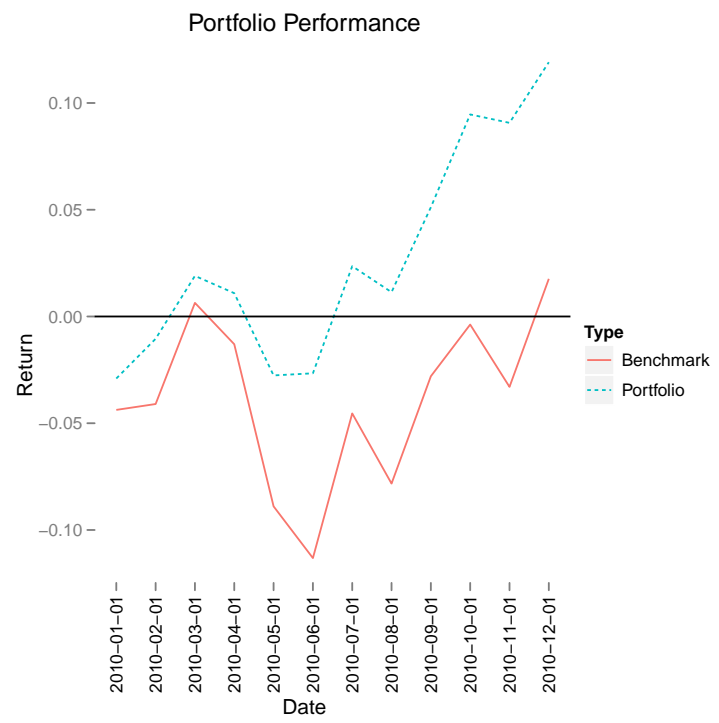

Figure 2: Performance Attribution.

methodology. Before reaching that level of complexity, however, pa provides a good starting point for basic performance attribution.

\section{Bibliography}

G. Brinson, R. Hood, and G. Beebower. Determinants of portfolio performance. Financial Analysts Journal, 42(4):39-44, Jul.-Aug. 1986. URL http://www. jstor. org/stable/4478947. [p53]

P. Carl and B. G. Peterson. PerformanceAnalytics: Econometric tools for performance and risk analysis, 2013. URL http: //CRAN. R-project. org/package=PerformanceAnalytics. R package version 1.1.0. [p53]

J. Enos and D. Kane. Analysing equity portfolios in R. R News, 6(2):13-19, MAY 2006. URL http: //CRAN.R-project.org/doc/Rnews. [p53, 60]

R. Grinold. Attribution. Modeling asset characteristics as portfolios. The Journal of Portfolio Management, page 19, Winter 2006. [p53]

T. Lord. The attribution of portfolio and index returns in fixed income. Journal of Performance Measurement, Fall:45-57, 1997. [p53]

J. Menchero. An optimized approach to linking attribution effects over time. Journal of Performance Measurement, 5(1):36-42, 2000. [p56]

J. Menchero, A. Morozov, and P. Shepard. The Barra Global Equity Model (GEM2). MSCI Barra Research Notes, Sept. 2008. [p53]

Morningstar Inc. Equity performance attribution methodology. Morningstar Methodology Paper, March. 2009. URL http://corporate.morningstar.com/us/documents/MethodologyDocuments/ MethodologyPapers/EquityPerformanceAttributionMeth. pdf. [p53]

Rmetrics Association. Rmetrics, 2013. URL https: //www. rmetrics.org/. [p53]

B. Singer and D. Karnosky. The general framework for global investment management and performance attribution. The Journal of Portfolio Management, Winter:84-92, 1995. [p53]

Yang $L u$

Williams College

2896 Paresky Center

Williamstown, MA 01267

United States

yang. lu2014@gmail.com

\author{
David Kane \\ Harvard University \\ IQSS \\ 1737 Cambridge Street \\ CGIS Knafel Building, Room 350 \\ Cambridge, MA 02138 \\ United States \\ dave.kane@gmail.com
}

